Revue d'Allemagne et des pays de langue allemande

50-2 | 2018

Humanités environnementales - Quoi de neuf du côté des méthodes?

\title{
„Critical Zones“ Ein Forschungsseminar mit Bruno Latour
}

Daniel Irrgang, Martin Guinard-Terrin et Bettina Korintenberg

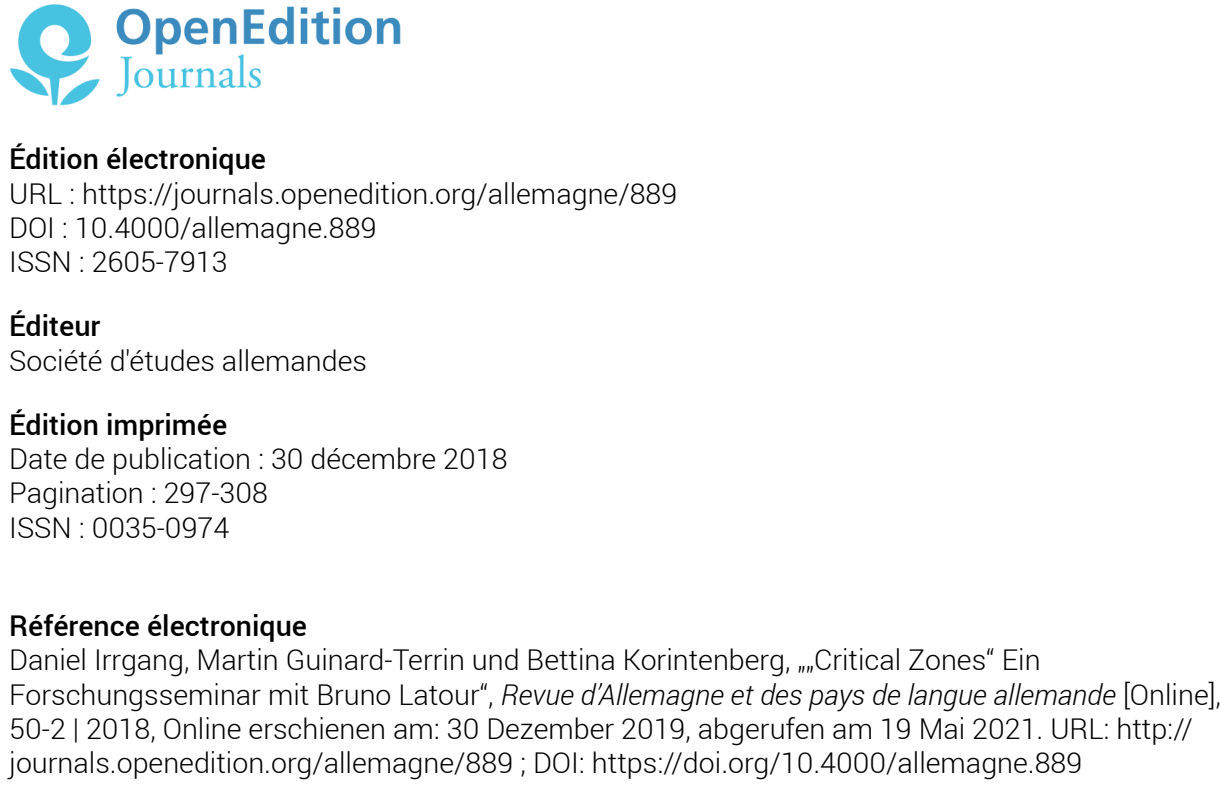

Édition électronique

URL : https://journals.openedition.org/allemagne/889

DOI : 10.4000 /allemagne.889

ISSN : 2605-7913

Éditeur

Société d'études allemandes

Édition imprimée

Date de publication : 30 décembre 2018

Pagination : 297-308

ISSN : 0035-0974

Référence électronique

Daniel Irrgang, Martin Guinard-Terrin und Bettina Korintenberg, "mCritical Zones" Ein

Forschungsseminar mit Bruno Latour", Revue d'Allemagne et des pays de langue allemande [Online] 50-2 | 2018, Online erschienen am: 30 Dezember 2019, abgerufen am 19 Mai 2021. URL: http:// journals.openedition.org/allemagne/889 ; DOI: https://doi.org/10.4000/allemagne.889 


\section{"Critical Zones“ Ein Forschungsseminar mit Bruno Latour}

- Daniel Irrgang*, Martin Guinard-Terrin **, Bettina Korintenberg ***

„Alles muss aufs Neue kartografiert werden“, fordert Bruno Latour in seinem unlängst erschienenen Terrestrischen Manifest ${ }^{(1)}$. Die französische Originalausgabe erschien 2017 bei Éditions La Découverte, Paris, unter dem Titel Où atterrir? Comment s'orienter en politique. Wesentlich besser noch als die deutsche Variante benennt dieser Titel die Leitfrage nicht nur des Essays, sondern auch die des Forschungsseminars, welches Latour zurzeit an der Staatlichen Hochschule für Gestaltung (HfG) Karlsruhe gibt: Wie orientieren?

Seit Januar 2018 entwickelt der französische Philosoph und Soziologe Bruno Latour als Gastprofessor an der HfG Karlsruhe experimentell eine imaginäre Kartografie, vermittels derer eine Orientierung gelingen mag in jenen zerrissenen Verhältnissen in Gesellschaft, Politik und Ökologie, die er als Neues Klimaregime ${ }^{(2)}$ bezeichnet hat. Klima wird in diesem erweiterten Sinne allgemein verstanden als die „Beziehungen der Menschen zu ihren materiellen Lebensbedingungen “(3). In mehreren Blockseminaren bereitet Latour zusammen mit Studierenden und Postgraduierten der Hochschule aus Theorie, Gestaltung und den Künsten sowie Forschern und Künstlern aus

* Wissenschaftlicher Mitarbeiter im Fachbereich Kunstwissenschaft und Medienphilosophie an der Staatlichen Hochschule für Gestaltung Karlsruhe.

$*$ Kunsthistoriker und Kurator. Zusammen mit Bruno Latour hat er die Ausstellung „Reset Modernity!“ mitentwickelt und ist zurzeit unter anderem Co-Kurator für das Projekt „Critical Zones“.

*** Kuratorin am ZKM (Zentrum für Kunst und Medien Karlruhe).

1 Bruno Latour, Das terrestrische Manifest, übersetzt aus dem Französischen von Bernd Schwibs, Berlin, Suhrkamp, 2018, S. 43.

2 Bruno Latour, Kampf um Gaia. Acht Vorträge über das neue Klimaregime, übersetzt aus dem Französischen von Achim Russer und Bernd Schwibs, Berlin, Suhrkamp, 2017. Französische Ausgabe: Face à Gaïa. Huit conférences sur le nouveau régime climatique, Paris, La Découverte, 2015.

3 B. Latour, Das terrestrische Manifest (Anm. 1), S. 9. 
verschiedenen Teilen der Welt ${ }^{(4)}$ konzeptionell eine Ausstellung vor, die am ZKM (Zentrum für Kunst und Medien Karlsruhe) zu sehen sein wird. In den Wochen zwischen den einzelnen Blockveranstaltungen arbeiten die Teilnehmer einzeln oder in Gruppen an Forschungsprojekten, die zur Konzeption der Ausstellung beitragen oder konkrete Arbeiten für sie entwickeln. Ergänzt werden die Positionen im Seminar durch Gäste, etwa aus den Geowissenschaften oder den Künsten. So nahm an der letzten Sitzung im Mai der renommierte Foto- und Filmkünstler Armin Linke teil, der nicht nur Ausschnitte aus seinen aktuellen Dokumentarfilmprojekten mit Bezug zum Thema des Seminars vorstellte, sondern die Teilnehmer auch dazu eingeladen hat, mit der großen Menge an Filmmaterial, das sein umfangreiches Archiv darstellt, $\mathrm{zu}$ arbeiten und thematische Verbindungen zu ihren individuellen Forschungsinteressen herzustellen. Armin Linke hat zudem die zweite Seminarsitzung fotografisch festgehalten - wir danken ihm für die freundliche Erlaubnis, hier zwei dieser Fotos abdrucken zu dürfen.

\section{Präsentation von Ergebnissen einer Gruppenarbeit im Rahmen der zweiten Sitzung des Forschungsseminars im Mai 2018 an der HfG Karlsruhe}

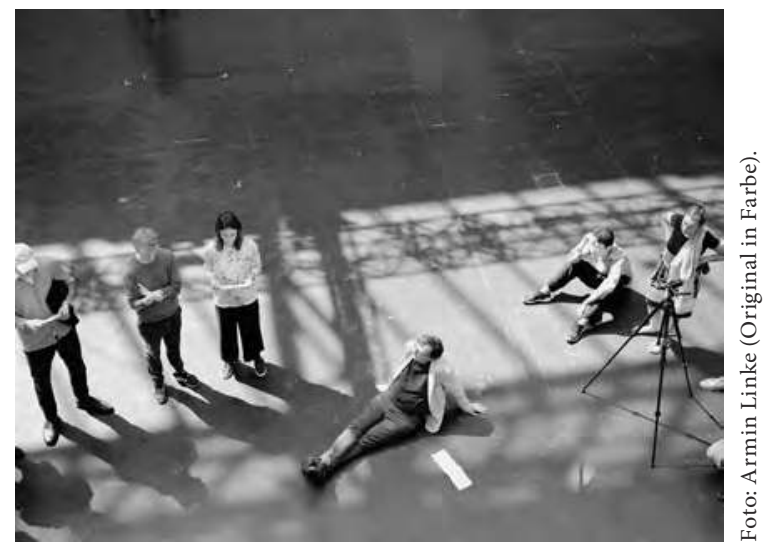

Die Konstellation einer Gastprofessur, die Studierende der HfG sowie weitere Beteiligte in ein Forschungs- und Ausstellungsprojekt am ZKM mit einbezieht, folgt der Strategie einer engeren Kooperation beider Häuser, wie sie zwischen den beiden Schwesterinstitutionen in ihren Gründungsjahren angelegt und gelebt wurde ${ }^{(5)}$. Als

4 Die 28 Teilnehmer stammen unter anderem aus Deutschland, Frankreich, Japan, Südkorea, Türkei und den USA. Die Autorin und die Autoren dieses Textes zählen ebenfalls zu den Teilnehmern und haben als Koordinator des Seminars für die HfG (Daniel Irrgang), Co-Kurator der Ausstellung (Martin Guinard-Terrin) und Mitarbeiterin im kuratorischen Team des ZKM (Bettina Korintenberg) weitere Einblicke in das Gesamtprojekt.

5 Unter dem Gründungsdirektor Heinrich Klotz, der damals noch beiden Institutionen in Personalunion vorstand, war Forschung, Lehre und Ausstellungsbetrieb von ZKM und HfG Karlsruhe als verschränktes System gedacht. „Auf diese Weise wird die künstlerische Selbstkontrolle an der vorderen Linie der Medienentwicklung und der Erschließung der Medienkünste unterstützt" (Heinrich KLOTz, Eine neue Hochschule (für neue Künste), Schriftenreihe der Staatlichen Hochschule für Gestaltung 
Modellprojekt diente die Entwicklung einer Ausstellung zum Leben und Wirken des mallorquinisch-katalanischen Gelehrten und Mystikers Ramon Llull. Amador Vega Esquerra, Professor für Ästhetik an der Universität Pompeu Fabra in Barcelona, bereitete im Wintersemester 2016/17 als Gastprofessor an der HfG die forschungsintensive Ausstellung zusammen mit Studierenden und Beteiligten beider Häuser vor. „DIA-LOGOS. Ramon Llull und die Kunst des Kombinierens“ ist seit 17. März 2018 am ZKM zu sehen (voraussichtlich bis zum 5. August 2018) und wurde kuratiert von Amador Vega Esquerra, Peter Weibel und Siegfried Zielinski, in Zusammenarbeit mit Bettina Korintenberg und Daniel Irrgang.

Einem ähnlichen Modell folgt das aktuelle Ausstellungsprojekt, kuratiert von Bruno Latour, Peter Weibel und Martin Guinard-Terrin: „Critical Zones“ wird 2020 am ZKM eröffnen, um die Merkmale des Neuen Klimaregimes zu kartografieren und die Ausstellungsbesucher mit den veränderten „terrestrischen“ Bedingungen in Beziehung zu setzen - Bedingungen, in denen wir zu leben erst noch lernen müssen $^{(6)}$. Latours Arbeiten zum Neuen Klimaregime stehen in direkter Beziehung mit jener „Anthropologie der Modernen“ ${ }^{(7)}$, die sich nicht nur in seiner Veröffentlichung der letzten Jahrzehnte herausgebildet hat ${ }^{(8)}$. Auch basieren sie auf Ausstellungsprojekten, die Latour in den letzten 20 Jahren in Kooperation mit dem ZKM entwickelt hat und die er und Peter Weibel, analog zum Begriff des Gedankenexperiments, als „Gedankenausstellungen“ bezeichnen: „Iconoclash“ (2002), „Making Things Public“ (2005) und „Reset Modernity!“ (2016). Ausstellungen also, die „die Erfahrung des Denkens und das Voranschreiten in einer bestimmten Form des Denkens “(9) mit der Erlebniswelt der Besucher konfrontieren. Als Ergebnis der Zusammenarbeit von Künstlern, Wissenschaftlern, Gestaltern sowie Aktivisten zeigen diese Gedankenausstellungen Brüche in jener Welt an, die wir als Moderne zu bezeichnen gelernt haben. Die künstliche Welt des Ausstellungsraums erlaubt es, mit den Krisen, die sich in diesen Brüchen zeigen, aber auch mit ihren Möglichkeiten, experimentell und ausprobierend umzugehen. Hierbei kann sich ein testendes, spielerisches Verhältnis zu den neuen Bedingungen artikulieren, wie es in der Welt „da draußen“ so nicht möglich wäre.

Doch zurück zu „Critical Zones“ und den Anliegen dieses Forschungs- und Ausstellungsprojekts. Der Begriff critical zone stammt aus den Geowissenschaften und der Biologie, wo er verwendet wird, um die dünne „Haut“ des Planeten Erde zu

Karlsruhe, Stuttgart, Cantz, 1995, S. 28). Selbstredend fanden Kooperationen zwischen beiden Häusern auch in den letzten Jahren statt; die Institutionalisierung von Kooperationsmöglichkeiten - wie etwa über solche Gastprofessuren und Ausstellungsprojekte - soll diese Komplementarität als großen Vorteil für beide Häuser jedoch noch stärker ausbauen.

6 Vgl. B. Latour, Kampf um Gaia (Anm. 2), S. 32f.

7 A.a.O., S. 15.

8 Besonders hervorzuheben sind hier Wir sind nie modern gewesen. Versuch einer symmetrischen Anthropologie, übersetzt aus dem Französischen von Gustav Roßler, Frankfurt am Main, Suhrkamp, 2008 und Existenzweisen. Eine Anthropologie der Modernen, Berlin, Suhrkamp, 2014; im Original erschienen als Nous n'avons jamais été modernes. Essai d'anthropologie symétrique, Paris, La Découverte, 1991 und Enquêtes sur les modes d'existence. Une anthropologie des Modernes, Paris, La Découverte, 2012.

9 Bruno Latour im Gespräch mit Heinz-Norbert Jocks, Kunstforum International, 237 (2015), S. 242. 
bezeichnen, seine Oberfläche, wenige Kilometer dick, auf der sich Leben so wie wir es kennen entwickelt hat und dort - und das ist zentral - die eigenen Bedingungen für sein Überleben hergestellt hat ${ }^{(10)}$. Diese Oberfläche ist fragil, angreifbar - kritisch -, aber somit auch hochgradig reaktionsfähig. Hier zeigt sich das mehrdeutige Potenzial des Begriffs critical zone, wie Latour ihn versteht: Er bezeichnet eine Perspektive auf die Biosphäre, die auf (menschliches) Einwirken höchst sensibel reagiert, die aber auch den Menschen als Teil und Bedingung in sich trägt.

\section{Typische Visualisierung der critical zone in den Geowissenschaften}

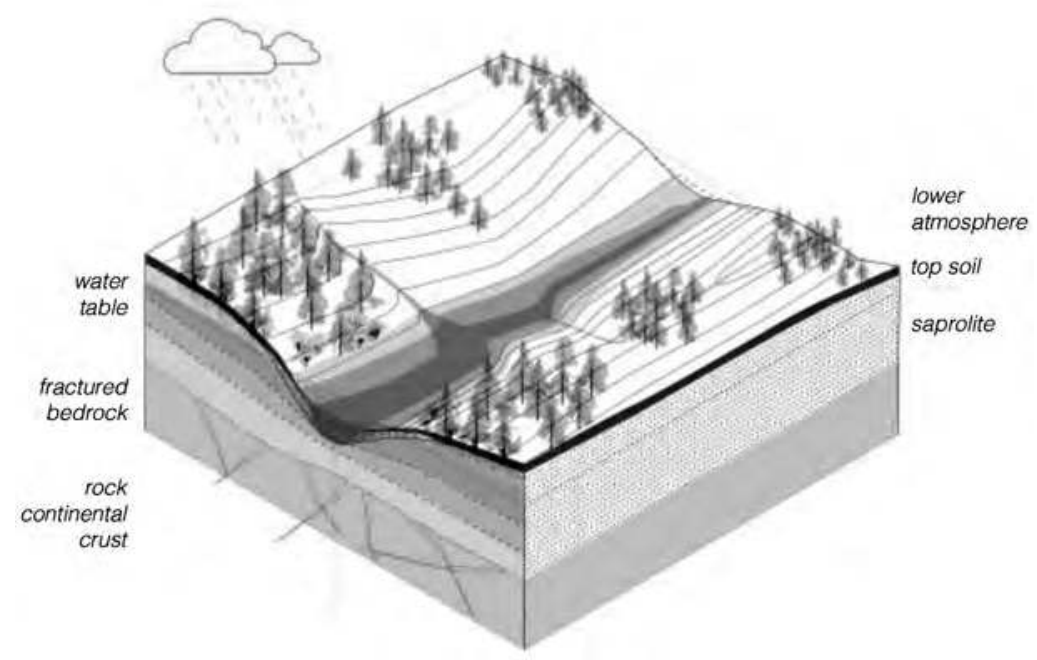

Abb. aus Alexandra Arènes, Bruno Latour und Jérôme Gaillardet, „Giving Depth to the Surface - an Exercise in the Gaia-graphy of Critical Zones", The Anthropocene Review, 2018.

Der alte Dualismus Kultur und Natur, das Einwirken des Menschen auf die Natur aus einer Stellung des Äußeren, wird aufgehoben zugunsten eines heterogenen Zusammenwirkens von Lebewesen und ihren geochemischen Bedingungen zu einem responsiven, sich selbst generierenden Zusammenhang, in den auch der Mensch als Teil eingebettet ist. Dies hat, folgt man Latour, philosophische Implikationen: Natur erscheint hier nicht als System, dessen ökologische Desaster es dadurch zu begegnen gilt, Elemente von Kultur möglichst verträglich zu integrieren. Solch eine Sichtweise, die jene der Moderne ist, impliziere eine mechanische, mitunter kybernetische Position, in der ein omnipräsenter „Ingenieur“ einen Überblick über dieses System einnehmen kann, um dessen vielfältige Bestandteile zu einem Ganzen zu integrieren ${ }^{(11)}$. Die Perspektive der critical zone hingegen ist keine konstruierte von außerhalb, keine

10 Vgl. Susan L. Brantley et al., „Designing a Network of Critical Zone Observatories to Explore the Living Skin of the Terrestrial Earth“, Earth Surface Dynamics, 5 (2017), S. 841-860.

11 Bruno Latour, „Some advantages of the notion of ,Critical Zone“ for Geopolitics“, Geochemistry of the Earth's Surface meeting, GES-10, Paris, France, Aug. 18-23, 2014, Procedia Earth and Planetary Science, 10 (2014), S. 3-6, hier S. 5. 
einer abstrakten „Natur“, die es distanziert zu beschreiben und begegnen gilt. Sie sieht den eigenen Standpunkt vielmehr als eingebettet, eingefaltet in eine Umwelt vielfältiger dynamischer Prozesse ${ }^{(12)}$. In der Entwicklung einer solchen Perspektive, die geochemische, geophysische sowie evolutionsbiologische Prozesse in den Fokus nimmt, spielen für Latour die (in den Fachdiskursen nicht unumstrittenen) Pionierleistungen von Lynn Margulis und James Lovelock eine zentrale Rolle ${ }^{(13)}$. Letzterer resp. sein Gaia-Konzept $^{(14)}$ ist ein wichtiger Bezugspunkt sowohl für Latours Buch Kampf um Gaia als auch für unser Forschungsseminar. Die Konferenz „Next Society - Facing Gaia“, die im April 2016 im Rahmen der Ausstellung „Reset Modernity!“ am ZKM stattfand und die die kritischen Bedingungen des Planeten Erde und die Frage, wie wir dort in Zukunft leben möchten, in den Blick nahm, kann als Auftakt des Critical Zones-Projekts verstanden werden.

Die Frage, die sich nun stellt ist, wie uns solch eine neue Sicht auf unsere Welt helfen kann, in einer Zeit globaler (sozialer, politischer, ökonomischer, ökologischer) Krisen ein neues Verhältnis zu ihr zu entwickeln; eines, das nicht mehr jenes abstrakte, distanzierte Verhältnis der Modernen ist, welches sich im Natur-KulturDualismus zeigt. In Das terrestrische Manifest kartografiert Latour diese Krisen und projiziert einen neuen Vektor für einen möglichen Weg in die Zukunft ${ }^{(15)}$. Folgt man seiner Gegenwartsanalyse (wir fassen sie hier nur skizzenhaft zusammen), so hat der Vektor der Moderne - die Globalisierung als Operator des Fortschrittsnarrativs ausgedient. Der Vektor als ein in die Zukunft offener Kurs ist zu einem definitiven Endpunkt geworden: Der Planet der Zukunft wird das Wachstum der Summe seiner Nationen sowohl kapazitär als auch ökologisch nicht mehr tragen können. Der Kurs nach vorn, Richtung Globalisierung, der dennoch im Schwung der Moderne noch immer vorangetrieben wird, ist un-realistisch geworden. Aber auch der Kurs zurück, in Richtung des Lokalen, der sich als eine Rückkehr zu Werten wie Heimat, Boden und Tradition gibt und Symptome wie Brexit und US-Präsident Trump generiert, existiert in den globalisierten Zusammenhängen nicht mehr. Was Latour fordert ist stattdessen ein Schritt „seitwärts“, in Richtung des „Terrestrischen“ - die Erde, auf der wir leben, weder als "globalisierten Globus“ verstanden, noch als Akkumulation von Territorien ihrer Nationalstaaten. Sondern als jenen interdependenten, sich selbst generierenden Zusammenhang, wie er oben in der Perspektive der Critical Zones beschrieben wurde: „Das Terrestrische stellt nicht länger allein den Rahmen menschlichen Handelns dar, es ist vielmehr Teil davon. Der Raum ist nicht mehr der mit ihrem Raster aus Längen- und Breitengraden erfasste der Kartografie, sondern ist zu einer bewegten Geschichte geworden, in der wir selbst nur Beteiligte unter anderen

12 Ebd.

13 Vgl. zu diesen Pionierleistungen Bruce ClARke, „Gaia is not an Organism - Scenes from the Early Scientific Collaboration between Lynn Margulis and James Lovelock“, in: Lynn Margulis. The Life and Legacy of a Scientific Rebel, hg. von Dorian SAGAn, White River Junction (VT), Chelsea Green, 2012, S. 32-43.

14 Vgl. u.a. James Lovelock, Gaia. The Practical Science of Planetary Medicine (1991), Oxford, Oxford University Press, 2000.

15 Vgl. hierzu auch Bruno Latour, „On a Possible Triangulation of Some Present Political Positions“, Critical Inquiry, 44/2 (2018), S. 213-226. 
sind, die auf Reaktionen anderer reagieren“(16). Die Aufgabe des Forschungsseminars ist es, diesen Möglichkeitsraum des Terrestrischen beschreibbar und schließlich als Ausstellung erfahrbar zu machen.

Das Forschungsseminar hat sich dieser Aufgabe gestellt ${ }^{(17)}$. Das interdisziplinäre Seminar, in dem sich Präsentationen Bruno Latours und der Beteiligten mit Diskussions- und Workshopformaten abwechseln, ist ein Versuch, sich den vielfältigen Implikationen der Critical Zones bzw. des Terrestrischen aus verschiedensten Perspektiven zu nähern, als ein Oszillieren zwischen Wissenschaften, Künsten und Politik.

Ähnliche Seminar-/Workshopformate im Kontext von Denkausstellungen wurden von Bruno Latour bereits erfolgreich durchgeführt, etwa in Shanghai im Kontext der Ausstellung „Reset Modernity!“ im April 2016, einer Einladung von Hans Ulrich Obrist und Yangwoo Lee folgend. Eine weitere Workshopreihe, die die Implikationen von „Reset Modernity!“ und „Critical Zones“ zusammenführt, findet zurzeit in Teheran statt, durchgeführt von Bruno Latour und Martin Guinard-Terrin an der Pejman Foundation, in Kooperation mit der Universität Teheran und dem ZKM. Die „Iran-Perspektive“ nimmt noch sensitiver als das Seminar in Karlsruhe die postkolonialen Implikationen einer Gegenwartsanalyse in Hinblick auf die Brüche mit der (europäischen) Moderne in den Fokus. In diesem Workshop stellen Künstler, Geografen, Geologen sowie Soziologen in Vorträgen Projekte vor, die in intensiven Diskussionsformaten analysiert werden. Im Verlauf des Workshops werden Dokumente der Teilnehmer gesammelt und an Präsentationswänden zur weiteren Bearbeitung bzw. zum Herstellen von Verbindungen zwischen den diversen Materialien montiert.

Das Seminar an der HfG Karlsruhe verfolgt eine etwas andere, stärker didaktische Herangehensweise. Es ist in Blöcken zu je fünf Tagen organisiert, die erste Sitzung fand im Februar 2018 statt, gefolgt von einer zweiten im Mai. Vier weitere werden bis Ende 2019 folgen. Thematisch ist es in drei Stränge strukturiert:

1. „Die Wissenschaft der Critical Zones“ - mit Unterstützung von Wissenschaftlern aus den Geowissenschaften, die an politischen und künstlerischen Fragen interessiert sind, vor allem aus den sogenannten Long Term Ecological Research (LTER)und Long Term Socio-economic and Ecosystem Research (LTSER)-Netzwerken in Frankreich und Deutschland. Jérôme Gaillardet, Geophysiker am französischen Institut de physique du globe, ist hier der wichtigste Partner und Berater.

2. „Die Kunst der Critical Zones“ - anhand der Werke von und durch die Zusammenarbeit mit Künstlern, die Praktiken verwenden, welche Bruno Latour als „material turn in the arts“ beschreibt. Hier bestanden bereits Kollaborationen unter anderem mit Toma's Saraceno, Joana Hadjithomas \& Khalil Joreige, Sophie Ristelhueber und Adam Lowe.

3. „Die Sozial- und Politikwissenschaften der Critical Zones“ - womit solche Positionen gemeint sind, die bereit sind, über Vorstellungen des Globalen hinauszugehen und über Alternativen nachzudenken (unter anderem Perspektiven mit Verbindungen zur Philosophie und Geographie).

16 B. Latour, Das terrestrische Manifest (Anm. 1), S. 53.

17 Die folgende Beschreibung des Seminarprinzips orientiert sich an einem internen Konzeptpapier Bruno Latours sowie an den Erfahrungen der Autorin und der Autoren als Teilnehmer am Seminar. 
Die Seminarteilnehmer nähern sich interdisziplinär der Herausforderung, das vermittels des Begriffs Critical Zones aufgespannte Diskursfeld nicht nur forschend zu erkunden, sondern die dabei entwickelten Ideen auch in den Erfahrungsraum einer Ausstellung zu übertragen, in dem nicht nur der logos, sondern auch Ästhetik, Affekt, räumliche Beziehung und soziale Interaktion eine Rolle spielen. Das Ziel ist es, für die Perspektive des Terrestrischen Repräsentationen und Narrationen zu finden, die einen Zugang der Öffentlichkeit ermöglichen. (Sie existieren noch nicht, im Gegensatz zu den vielen machtvollen Repräsentationen und Narrationen des Globalen und Lokalen.)

Eine der Methoden, die hierfür im Seminar angewendet werden, ist das Versetzen der Teilnehmer in imaginäre Situationen, die Merkmale von Gedankenexperimenten tragen. Solch ein Experiment, auf das die Teilnehmer im Verlauf des Seminars immer wieder zurückkommen, fand in der ersten Sitzung statt ${ }^{(18)}$ : Angenommen wir leben um 1610 und erfahren von den revolutionären Entdeckungen Galileo Galileis. Nun ist man vor die Herausforderung gestellt, die dadurch hereinbrechenden grundlegenden Veränderungen für die Kosmologie und Gesellschaft dieser Zeit zu erkennen und zu verarbeiten. Als Ausgangspunkt bzw. Denkmaterial diente uns Bertolt Brechts episches Theaterstück Leben des Galilei (uraufgeführt 1943). Das Gedankenexperiment unternimmt sodann eine Zeitreise: Angenommen wir befinden uns wieder im Jahr 2018 und sind mit ähnlich grundlegenden Umbrüchen in Kosmologie und Gesellschaft konfrontiert, wie wir sie mit der neuen Perspektive des Terrestrischen assoziieren. Wie würde man mit vergleichbaren epistemischen Strategien mit diesen Umwälzungen fertig werden? Um die Parallele zwischen 1610 und 2018 greifbarer zu machen, ersetzen wir Galileo Galilei mit James Lovelock und seiner „Entdeckung“ des Planeten Erde als responsive Quasi-Lebensform, die er mit der Denkfigur Gaia zu fassen versucht. Die Attraktivität, solche (absichtlich historisierenden) Parallelen aufzustellen und zu analysieren, besteht darin, mit Mitteln der Wissenschaftsgeschichte, Kunstgeschichte, Philosophie, Kartografie, der Künste usw. die Merkmale beider epistemischer Brüche zu identifizieren und die Seminarteilnehmer auf die Parallelen zwischen diesen beiden historischen Ereignissen (denn als solches bezeichnen wir auch, mit Latour, Lovelocks Entdeckung) in eigener Denkarbeit aufmerksam zu machen. Neben den Parallelen interessieren uns aber auch die Unterschiede, nämlich der bereits erwähnte Mangel an adäquaten Repräsentationen des Neuen Klimaregimes, die dessen Implikationen den Menschen näherbringen könnten. Folgt man Latour, so herrschte in der wissenschaftlichen Revolution des 17. Jahrhunderts eine wesentlich stärkere Kontinuität in den Repräsentationen und Narrationen des Kosmos vor und nach Galilei: „No sooner had the Copernican system been proposed, images of the Sun King began to appear in Versailles...“(19). Für die Umbrüche, die wir mit dem Neuen Klimaregime und der Critical Zones-Perspektive zu analysieren versuchen, sieht die Situation völlig anders aus. Diese neue „Kosmologie“ wird keinesfalls ohne Weiteres akzeptiert, wie uns etwa die immer lauter werdenden Stimmen der Klimaleugner zeigen, mit dem aktuellen US-Präsidenten als ihren Advokaten. Gehen wir jedoch davon aus, dass es

18 Die folgende Beschreibung basiert auf einem Konzeptpapier Bruno Latours (Februar 2018).

19 Ebd. 
sich bei dem Neuen Klimaregime um eine mindestens genauso signifikante Revolution wie jene im 17. Jahrhundert handelt, wird der Bedarf an adäquaten Repräsentationen und Narrationen zentral. Hierin liegt die Motivation Bruno Latours begründet, zusammen mit jungen Künstlern, Wissenschaftlern und Aktivisten im geschützten Raum eines experimentellen Forschungsseminars die Potentiale der Critical Zones zu erkunden. "Such a collaboration is indispensable to generate another landscape where it is possible to give another orientation for political involvement ${ }^{\text {“(20) }}$.

\section{Gruppenarbeit im Rahmen der zweiten Sitzung des Forschungsseminars im Mai 2018 an der HfG Karlsruhe}

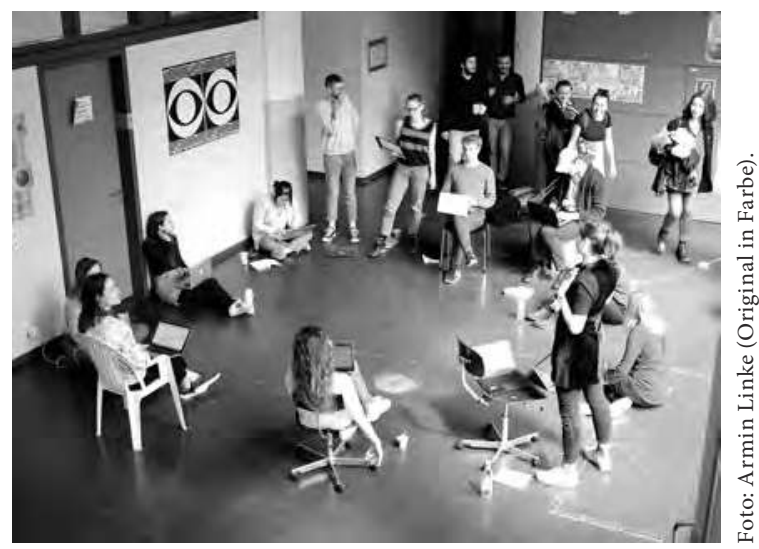

Als Artikulation für die im Forschungsseminar durchgeführten kollektiven Gedankenexperimente haben sich die Methoden des Reenactments und Rollenspiels bewährt. So wurden etwa die Protagonisten von Brechts Leben des Galilei in die Zukunft transportiert und der Hauptdarsteller durch Lovelock ersetzt, wobei es nicht lediglich um eine historische Analogie ging, sondern um ein Erforschen der Protagonisten jener Diskurse und ihrer, um Latours bekannten Akteurs-Operationsmodus ins Spiel zu bringen, agency: Welche Interessen und Positionen treffen mit welcher Handlungsmacht aufeinander, um Status und Gültigkeit neuer epistemischer Konstellationen zu verhandeln? Besonders hervorheben möchten wir ein weiteres solch performatives Gedankenexperiment, welches in der zweiten Sitzung im Mai 2018 durchgeführt wurde. In verschiedene Gruppen aufgeteilt, versetzten sich die Teilnehmer in die Rolle von „Einwohnern“ einerseits des alten Territoriums, des Nationalstaats, und andererseits des neuen terrestrischen Raumes. Die beiden Parteien sollten nun ein erstes Aufeinandertreffen simulieren und einen Weg finden, wie sie ihre unterschiedlichen Verhältnisse zur Welt einander vermitteln können. Die Leitkategorien, die es der jeweils anderen Seite näher zu bringen galt, waren die Identität der Gemeinschaft (demos), die Konstruktion der jeweiligen Gottheit resp. des religiösen Systems (theos) und die sich daraus ergebende Kosmologie (cosmos). Zugegeben, dies 
sind abstrakte Kategorien, die nicht leicht in performativer Interaktion zu vermitteln sind. Die verschiedenen Gruppen fanden dennoch bemerkenswerte Strategien, sich einander anzunähern. Hier soll beispielhaft ein Auszug aus dem Ergebnis der Arbeit einer Gruppe, selbstbetitelt als „Anonymous Terrestrial Meeting“, gezeigt werden, die die Form eines abwechselnd vorgetragenen, zum Teil ins Polyphone wechselnden Manifests angenommen hatte. Im folgenden Auszug spricht das Manifest aus der Perspektive des theos:

„Believing that it's better not to believe, in contradiction.

I believe in the terrestrial, the possibility of chance, while my divinity is the future I seek for. My limits are the ones I oversee and a certain self-evolving power from the multiplicity of interconnected parts.

Religion is caring."

„My divinity is becoming, the possibility to move. The luxury to explore the world in a playful and energizing way and meeting strangers or talking to a stranger to understand others who are not in your inner circle.

Everyone is collective.

Being afraid of something is setting yourself a limit."

„Having a set of values that orient judgement and vision, a point of reference that links me to the Other on a less tangible, human level.

,Taking care not only of the self, but of what surrounds me, individual-collective responsibility, allowing for doubts and contradictions."

„A daily routine, a moving mind, the feeling of being a part of a community.

friendship/family

health/taking care

to exchange, to learn

I believe in the never ending experimental flow of life."

"Maybe I forgot where I wanted to go and now I am also not sure where I should go."

„Knowing that there will be an end of life - my life is not endless - leads me to experiment.

I feel like happiness and satisfaction might stop me."

Die Ergebnisse solcher Vorträge wurden im Anschluss verglichen und diskutiert. Im Fall des Manifests wurde dessen poetische Kraft als spekulatives Potential für eine "tastende" Herangehensweise an die noch undeutliche Situation einer Begegnung zwischen Vertretern des Territoriums mit Vertretern des Terrestrischen hervorgehoben.

Ein weiterer wichtiger Teil des Forschungsseminars sind jene Aufgaben, die zwischen den einzelnen Sitzungen bearbeitet werden. So entwickelten die Teilnehmer zwischen Februar und Mai erste Skizzen für eigene Forschungsprojekte, die im Rahmen der Laufzeit des Seminars individuell oder in Gruppen bearbeitet werden und gegebenenfalls direkt zur Ausstellung beitragen können. Die in der Mai-Sitzung präsentierten Projektskizzen waren so vielversprechend wie vielfältig; sie reichen von Recherchen zum Ausstellungsformat, über theoretische und historische Beiträge, bis hin zu künstlerischen Arbeiten. Zur letzten Kategorie gehört die hier exemplarisch gezeigte Arbeit von Michail Rybakov, Alumnus des Studiengangs Medienkunst an der HfG Karlsruhe; sie ist nur eine von mehreren Ideen, die er im Seminar vorgestellt hatte. Die Arbeit geht von den taxonomisch-klassifikatorischen Bildtafeln 


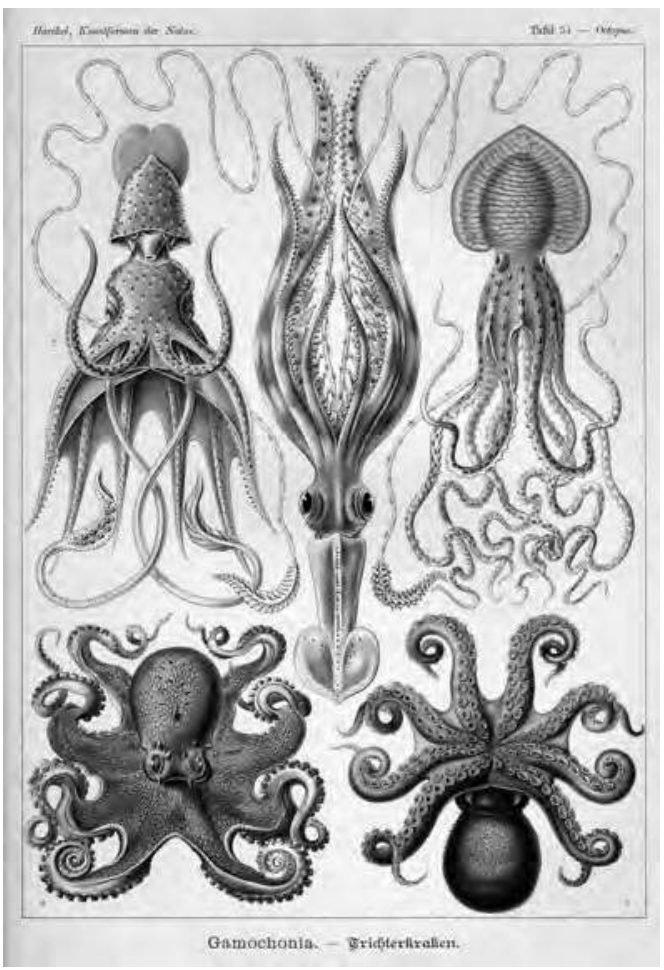

Beispiel für eine der verwendeten Bildtafeln: Tafel 54, „Gamochonia“, aus Ernst Haeckels Kunstformen der Natur

(Komplettausgabe, Leipzig/Wien, Bibliographisches Institut, 1904 - Original in Farbe).

Ernst Haeckels (1834-1919) aus, der als Zoologe wie kaum ein anderer den sogenannten westlichen Geist der Moderne auf eine externe Beobachterposition auf die Natur „von außen“ eingestimmt hat - sein berühmter Stammbaum des Menschen (1874) sieht bekanntlich den Menschen als Baum-Krone der Schöpfung vor. Rybakov bearbeitete nun diese Bildtafeln, und zwar nicht mittels des Genius des Künstlers und seiner Imagination, sondern vermittels der technischen „Einbildungskraft“ von neural networks, vermittels Bildprozessierung durch künstliche Intelligenz. Über deep learning-Algorithmen wurde das Programm mit Haeckel-Bildtafeln gefüttert und darauf trainiert, visuelle Muster zu erkennen. Daraus wurden schließlich neue Bilder generiert, indem der illustrative Stil Haeckels auf solche Schnittdiagramme aus den Geowissenschaften projiziert wird, wie jenes das im hier vorliegenden Text zur Illustration der critical zone verwendet wird. Herausgekommen sind Bildwelten, die uns seltsam vertraut, aber dennoch faszinierend fremd erscheinen. Sie zeigen skurrile "Landschaften" von ineinander gefaltetem biologischem Material - wie Lebenswelten, die ihre eigene Umwelt selbst generieren und die uns an jene Perspektive auf die Biosphäre erinnern, wie wir sie als critical zone zu lesen gelernt haben. Rybakov selbst über seine Arbeit: „We are conditioned to look for meaning in scientific illustrations. While the meaning is lost through the process of neural style transfer, the illustration gets a lot richer visually, and is just as interesting to examine“(21).

Nach diesem kleinen Schulterblick auf die beiden ersten Sitzungen des Forschungsseminars, und in Antizipation der vier noch kommenden, lässt sich vorläufig festhalten, dass dessen offener Rahmen eine der übergeordneten Forschungsfrage adäquate Art der Annäherung zu sein scheint. Diese Frage - Wie orientieren? - ist ebenso offen formuliert und auch von Bruno Latour selbst nicht beantwortet. Das Seminar nähert sich der Frage explorativ, mit experimentellen, mitunter phänomenologisch und performativen Mitteln. Der solide Input, der durch die einzelnen Vorträge Bruno Latours gegeben wird - „Bruno Latour beim Denken zuschauen“ ist

21 https://rybakov.com/blog/visualizing_complexity/. 
uns als faszinierte Rückmeldung der Teilnehmer immer wieder begegnet -, in Kombination mit dem offenen Rahmen für Diskussionen, Reenactments, Performances, Gruppenarbeit und individuelle Forschungsarbeit, bietet die Vorzüge der Kombination aus „vertikalem“ Unterricht und „horizontalem“ Austausch mit Latour in einer flachen Hierarchie. Nicht der Weg oder gar das Denk-Ziel sind vorgegeben, sondern skizziert werden Bedingungen, Symptome und Merkmale der Critical Zones, um davon ausgehend einen Möglichkeitsraum zu kartografieren, in dem - vielleicht adäquate Narrationen und Repräsentationen des Neuen Klimaregimes entwickelt werden können.

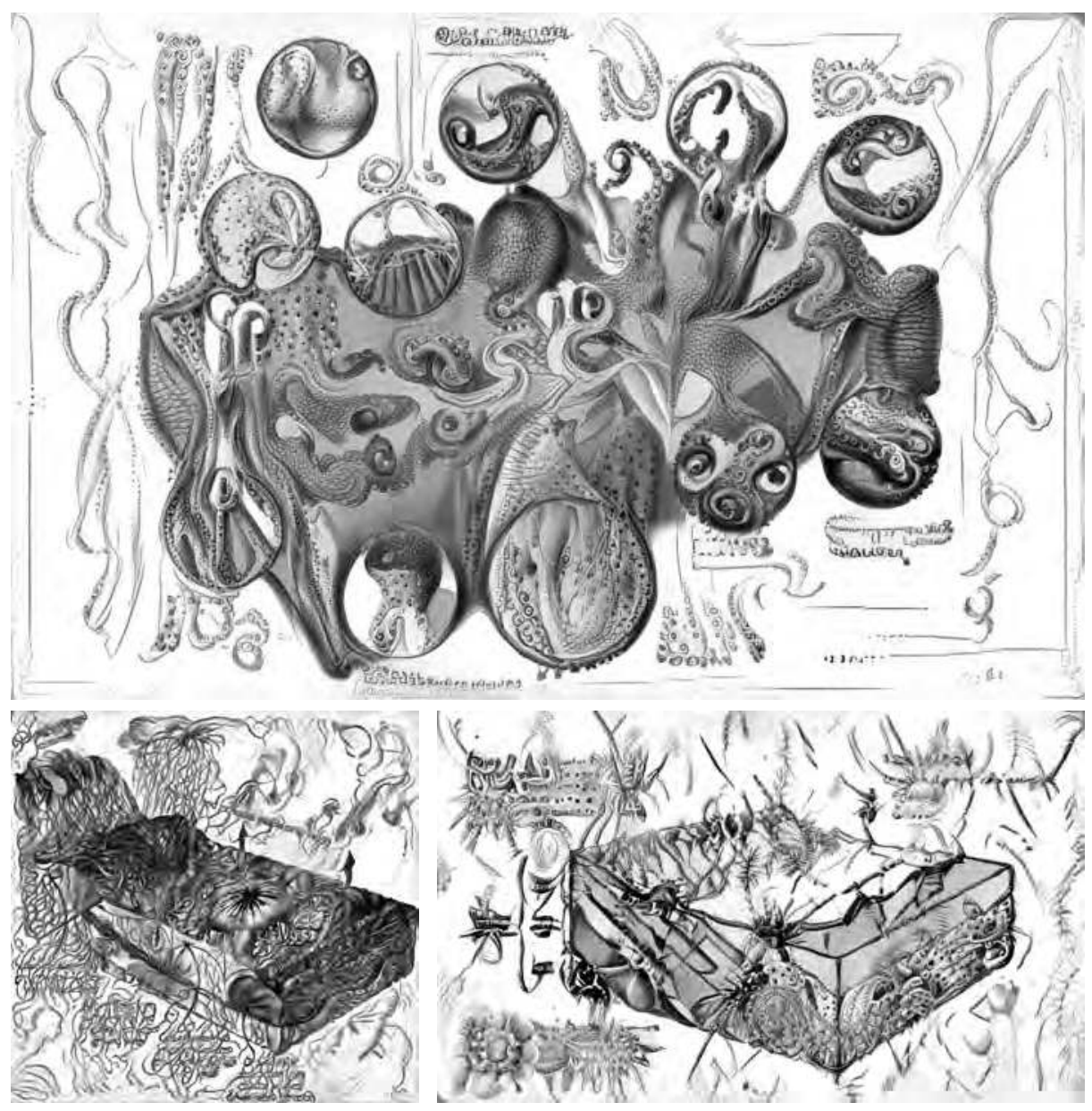

Ergebnis des neural style transfer algorithm, Michail Rybakov, 2018. 


\section{Zusammenfassung}

In einem Forschungsseminar an der Staatlichen Hochschule für Gestaltung Karlsruhe bereitet Bruno Latour zusammen mit den Teilnehmern aus Wissenschaften und Künsten die Ausstellung „Critical Zones“ vor, die 2020 am ZKM (Zentrum für Kunst und Medien Karlsruhe) eröfnen wird. Sowohl das Forschungsseminar als auch die Ausstellung befassen sich mit dem geophysikalisch kritischen Zustand der Erde, mit der sogenannten critical zone, ihrer durchlässigen, oberflächennahen Schicht. Von Bruno Latour wird der Begriff erweitert zu einem kritischen, teilnehmenden Verhältnis zu unserer Lebenswelt, deren bedrohter Zustand in der vom Menschen geprägten Erdgeschichte ein noch nie dagewesenes Ausmaß erreicht hat und dessen historischen Zusammenhang er als Neues Klimaregime beschrieben hat. Der Artikel versteht sich als Bericht aus diesem explorativen Forschungsprozess, erstellt von den kuratorischen Mitarbeitern des Projekts sowie dem Koordinator des Seminars, mit Fokus auf Erkenntnisinteressen und Methodologie. 